\title{
Interactive comment on "Ventilation of the Northern Baltic Sea" by Thomas Neumann et al.
}

\section{Thomas Neumann et al.}

thomas.neumann@io-warnemuende.de

Received and published: 26 November 2019

First of all, we would like to thank the anonymous referee for the thorough review of our manuscript.

The common concern of all referees is that the amount of data collected during a cruise in the sea ice covered northern Baltic Sea is not sufficient to justify any of the hypotheses raised in the manuscript. Indeed, only three stations could be explored and we hardly can increase the number of in-situ observations. However, the referees proposed an option to save the manuscript by a complementary modeling approach.

We decided to follow this line and will perform a model simulation for the winter season 2016/2017. We will set up a model for the Baltic Sea with earmarked water masses allowing us to identify the origin of water which eventually arrives in the deep water of the Bothnian Bay. Nevertheless, we want to stress that recent ocean models are 
not able to reproduce a possible haline convection due to brine release. However, this approach will prolong a revised version of our manuscript and will include substantial OSD changes.

In the following, we respond to the referee's specific remarks. Remarks are shown and followed by our response.

Interactive

comment

Review \#1:

1.) Abstract. The abstract is not sufficiently informative. Basically, there is just one sentence, which describes, what this paper is about. I would suggest to exclude all general wording about the Baltic Sea, but to add more about the essence of this paper.

We will follow the suggestion and update the abstract in the revised version when the model simulations have been completed and evaluated.

2.) Page 2, lines 2-3: I would suggest to add more detailed explanation (not just reference on Peterson, 2018), how melting sea ice can produce considerable salt fluxes into the ocean? This is quite a new knowledge, and it is important to explain it in more details.

An extended explanation will be done in the revised version.

3.) Page 2, lines 3-4: The references to Aagaard,et al., (1981) and Skogseth et al. (2008) are not relevant in this context. These papers describe specific events of dense water formation and do not consider general theory of this event at all. Taking into account that the authors are considering horizontal advection, as the most probable mechanism of the surface water densification in the Bothnian Bay, I would suggest them to read papers by Shapiro et al (2003) and Ivanov et al. (2004), which summarized all known mechanisms of dense water formation and cascading (not only in the Arctic, but worldwide).

We are grateful for the literature recommendation. 
4.) Page 15, lines 19-20: "In areas shallower than the pycnocline, dense water can accumulate at the sea floor and form density driven plumes guided by topography" This is very speculative statement, which is not confirmed by the provided measurements, but only by the references to the older studies, where this idea was also rather claimed, but not strongly supported.

The referee is right. In a revised version, we will formulate this statement as a hypoth-

eractive esis which cannot be rejected a priori.

Interactive comment on Ocean Sci. Discuss., https://doi.org/10.5194/os-2019-48, 2019. 